\title{
Repetição de gravidez na adolescência: estudos sobre a prática contraceptiva em adolescentes
}

\section{Repeat pregnancy in adolescence: study about the practice of contraception in adolescents}

\section{Camomila Lira Ferreira*}

Universidade Federal do Rio Grande do Norte - UFRN, Natal, Rio Grande do Norte, Brasil

\section{Liliane Pereira Braga**}

Universidade Federal do Rio Grande do Norte - UFRN, Natal, Rio Grande do Norte, Brasil

\section{Ádala Nayana de Sousa Mata*** \\ Universidade Federal do Rio Grande do Norte - UFRN, Natal, Rio Grande do Norte, Brasil}

\section{Caroline Araújo Lemos****}

Universidade Federal do Rio Grande do Norte - UFRN, Natal, Rio Grande do Norte, Brasil

\section{Eulália Maria Chaves Maia*****}

Universidade Federal do Rio Grande do Norte - UFRN, Natal, Rio Grande do Norte, Brasil

\begin{abstract}
RESUMO
A gravidez na adolescência é um período crítico que traz riscos não apenas para a mãe, mas também, para o seu bebê. Tais riscos podem ser potencializados pela repetição de gravidez, constituindo-se como problema de saúde pública. Objetivou-se considerar o conhecimento e o uso de métodos contraceptivos entre 50 adolescentes, com idade média de 17 anos e com repetição de gravidez, atendidas em enfermarias da Maternidade Escola Januário Cicco, em Natal/RN/BR. Trata-se de um estudo do tipo descritivo-exploratório, com a utilização de questionário semi-estruturado. A análise dos dados foi realizada com base na estatística descritiva através da análise das frequências das respostas das adolescentes. Resultados mostram que $44 \%$ das adolescentes conhecem pelo menos 3 métodos contraceptivos, sendo a camisinha o método mais utilizado na primeira relação $(78,3 \%)$ e a pílula o mais utilizado no período que antecedeu a atual ou última gestação (68\%). Há conhecimento dos métodos e relato do uso, mas a repetição de gravidez evidencia a necessidade de estimular práticas contraceptivas mais eficazes que considerem as peculiaridades da adolescência.
\end{abstract}

Palavras-chave: Recorrência de Gravidez na Adolescência, Métodos Contraceptivos, Comportamento Contraceptivo. 


\begin{abstract}
Pregnancy adolescence presents risks not only to the mother, but also to her baby, which may be increased by repeat pregnancy resulting in health policy problems. This study intended to consider the knowledge and usage of contraception amongst 50 repeat pregnancy adolescents whose average age was 17 years in Natal/RN/BR, during their prenatal follow up in nurseries of the Januário Cicco maternity hospital. This is an exploratory-descriptive study that was executed by means of a semi-structured questionnaire. Data analysis was performed by descriptive statistics through frequency response analysis. Results showed that $44 \%$ of these adolescents were aware of at least 3 types of contraception methods, of which condoms were the most utilized during first sexual intercourse $(78,3 \%)$, with the oral contraceptive pill most used during the period prior to the current or last pregnancy $(68 \%)$. Despite the knowledge about these methods and also usage reports by adolescents, repeat pregnancy demonstrates a need for more efficient encouragement of contraception practice.
\end{abstract}

Keywords: Recurrence of Pregnancy in Adolescence, Contraception Methods, Contraception Practice.

\title{
1 I ntrodução
}

A adolescência é definida com base etária (dos 10 aos 19 anos) e por características inerentes a este período como as grandes transformações físicas, psicológicas e sociais (AQUINO et al., 2003). É um período de transição da infância para a idade adulta, mas também uma fase do desenvolvimento na qual se refere à imaturidade e à suposição de invulnerabilidade dos adolescentes. Além disso, associa-se à realização de comportamentos de risco para sua saúde, tais como o uso abusivo de drogas, as práticas sexuais desprotegidas que aumentam as chances de contrair doenças sexualmente transmissíveis (DSTs) e/ou de ocorrer gestações não planejadas (AQUINO et al., 2003; BELO; PINTO E SILVA, 2004; CÂMARA; SARRIERA; CARLOTTO, 2007; CHALEM et al., 2007; GOLDENBERG, P.; FIGUEIREDO, M. C. T.; SILVA, R. S. 2005; LIMA et al., 2004). Diante disso, e considerando que a gravidez é um período crítico de transição que faz parte do processo de desenvolvimento humano (MALDONADO, 1997), a gravidez na adolescência seria duplamente crítica: a crise da adolescência soma-se à crise da gravidez (OLIVEIRA, 1999). Tal afirmação pode ser compreendida considerando-se que ambas, a gravidez e a adolescência, são transições existenciais consideradas situações de crise, uma vez que implicam em mudanças complexas e exige adaptações, reajustamentos interpessoais, intrapsíquicos, assim como mudança de identidade (MALDONADO, 2000).

A gravidez entre jovens de 15 a 19 anos vem crescendo a cada ano, passando a ser considerada, atualmente, no Brasil e em outros países em desenvolvimento, como um problema de saúde pública, tendo em vista suas consequências na vida da adolescente (afastamento da 
escola e do mercado de trabalho), bem como para a sociedade (elevados índices de mortalidades, morbidade e altos custos). É frequente em todos os níveis sociais, porém assumindo maior gravidade nos níveis sócio-econômicos mais baixos (CARNIEL et al., 2006). Essa constatação traz inúmeras implicações para a questão da gravidez na adolescência e sua multifatoriedade, tendo em vista os diversos riscos e efeitos negativos à jovem mãe e seu bebê (CARNIEL et al, 2006; MICHELAZZO et al., 2004; SABROZA et al., 2004). Vale salientar que, apesar de ser considerado um "problema social", é preciso considerar que, para algumas adolescentes, a gravidez surge como alternativa para um projeto pessoal, já que sua ocorrência muitas vezes é dada num contexto marcado por oportunidades restritas, poucas opções de vida e uma trajetória de fracasso escolar (PANTOJ A, 2003).

Dados fornecidos pelo DATASUS para o estado do Rio Grande do Norte, por exemplo, apontam que, no ano de 2004, dos 13.424 nascidos vivos, $24,6 \%$ eram filhos de mães com idade entre 10 e 19 anos. Além disso, dentre as internações hospitalares realizadas no ano de 2005 no estado, 70,1\% ocorreram por motivo de gravidez, parto e/ou puerpério de adolescentes com faixa etária entre 15 e 19 anos. Tais informações têm despertado grande interesse, tanto de pesquisadores, quanto no âmbito das políticas públicas na adolescência.

Com isso, a gravidez na adolescência configura-se como uma questão de grande relevância, e, portanto, diversos estudos investigam os possíveis fatores relacionados a este fenômeno. Características próprias da adolescência como a impulsividade, o imediatismo, os sentimentos de onipotência e de indestrutibilidade, a idade precoce da menarca e da iniciação sexual, o uso inconsistente de métodos contraceptivos, a baixa autoestima e a deficiência de programas de assistência ao adolescente são alguns dos fatores responsáveis pelo aumento do número de adolescentes grávidas (BARRERA; LIMA et al., 2004; PICK, 2006; SABROZA et al., 2004; PERSONA; SHIMO; TARALLO, 2004).

A ausência de informações sobre sexualidade e fisiologia reprodutiva também é identificada como um fator relacionado à gravidez na adolescência, assim como a liberação sexual da sociedade e a influência dos meios de comunicação (que muitas vezes apresenta realidades diferentes daqueles vivenciadas pela maioria das famílias brasileiras), além da ideologia da maternidade (sonho de ser mãe), das carências emocionais da adolescente (apoio social insuficiente) e da ausência de projetos pessoais com os quais a maternidade pudesse interferir (BELO; PINTO E SILVA, 2004; OLIVEIRA, 1999).

Outros autores (GODINHO et al., 2000) discutem a importância de direcionar a atenção para a gravidez na adolescência, já que este fenômeno multifatorial nem sempre é um evento único e fortuito, 
visto que para algumas jovens ele acaba se repetindo. Estudos buscaram compreender os aspectos que levam à repetição de uma gestação nessa fase do desenvolvimento, na medida em que, como afirmam Persona et al. (2004), além dos fatores relacionados a uma única gravidez na adolescência, existem outros fatores que se relacionam com a repetição da gravidez na adolescência, como, por exemplo: a ausência de ocupação remunerada da adolescente, a baixa renda familiar, o envolvimento com parceiros mais velhos, residir com o parceiro, e ter um parceiro fixo.

Se a gravidez na adolescência, enquanto evento único, já traz uma série de elementos de risco para a jovem mãe, a sua repetição pressupõe consequências mais graves para a adolescente, tais como o pequeno intervalo entre os partos e a maior possibilidade de nascimento de um bebê com baixo peso; além de problemas como a sobrecarga imposta a essas garotas que têm que cuidar de dois ou três filhos e, muitas vezes, da casa e do companheiro (GODINHO et al., 2000).

Atualmente, há uma escassez de dados nacionais, regionais ou mesmo locais acerca da incidência da repetição de gravidez na adolescência, sendo necessário que outros estudos sejam desenvolvidos nesse âmbito. Dos estudos existentes, um recente foi realizado na Maternidade Municipal Mãe Esperança em Porto Velho/RO, revelou que $70,77 \%$ das adolescentes estudadas tiveram 0 primeiro filho ainda adolescente e destas $37,66 \%$ tiveram mais um, dois e até três filhos antes de completarem 20 anos (MONTEIRO, 2009). Os referidos dados são corroborados pela Organização Mundial da Saúde (OMS), o qual estima que $40 \%$ das adolescentes que engravidam vão ter uma segunda gestação nos três próximos anos (MONTEIRO, 2009).

Analisando o comportamento sexual das adolescentes, Longo (2002) observou que os fatores que exercem influência no início da vida sexual da jovem implicam numa série de consequências para seu comportamento sexual futuro, acreditando ser pouco provável que haja mudanças significativas no comportamento contraceptivo de uma jovem. A decisão mais importante em relação às suas práticas contraceptivas, por exemplo, é tomada no início de sua vida sexual. Este aspecto também é considerado por Sabroza et al. (2004), que conclui, em seu estudo, que o padrão gestacional é geralmente definido na adolescência, de modo que quanto mais jovem for a mãe, maior a probabilidade de multiparidade.

Nesta perspectiva, destaca-se a importância de estudar o comportamento sexual e reprodutivo das adolescentes através do conhecimento e uso de métodos contraceptivos por parte destas, uma vez que o uso correto e o conhecimento adequado destes métodos podem contribuir para a redução do índice de repetição de gestações na adolescência, a redução do número de filhos nesta fase 
de desenvolvimento e da incidência de doenças sexualmente transmissíveis (LONGO, 2002). Diante disso, vislumbrou-se a necessidade de investigar acerca do conhecimento e do uso de métodos contraceptivos entre adolescentes com repetição de gravidez, tenha(m) sido a(s) gestação(ões) anterior(es) levada(s) a termo ou não.

\section{Métodos}

A amostra deste estudo foi constituída, aleatoriamente, por 50 adolescentes puérperas internadas em enfermarias da Maternidade Escola Januário Cicco (MEJC), vinculada a Universidade Federal do Rio Grande do Norte (UFRN), em Natal/RN/BR, no período de janeiro a julho de 2007. Referência em alta complexidade para o Rio Grande do Norte, a Maternidade é considerada a mais importante maternidade do Estado (WIKIPÉDIA, 2010). O fator de aleatoriedade foi realizado a partir do interesse e voluntariedade das adolescentes com repetição de gravidez usuárias dos serviços da MEJ C, as quais eram abordadas aleatoriamente e questionadas sobre a disponibilidade em participar da pesquisa. Não foi possível realizar o cálculo amostral, visto que a instituição não possuía os dados quantitativos do índice de parturientes adolescentes, assim como da incidência de repetição de gravidez na adolescência. Na presente pesquisa, a adolescente tinha que atender aos seguintes critérios de inclusão: concordar em participar da pesquisa, ter entre 12 e 18 anos, segundo a definição de adolescente prevista pelo Estatuto da Criança e do Adolescente, e apresentarem repetição da gestação, tenha(m) sido a(s) anterior(es) levada(s) a termo ou não.

Para a coleta de dados, foi utilizada a técnica da entrevista estruturada em situação individual nas enfermarias da MEJC. Antecedendo a aplicação do instrumento, foi informado adequadamente à adolescente e sua responsável sobre os objetivos da pesquisa, aplicação do instrumento, privacidade, e destino dos dados; e foi obtido o consentimento livre e esclarecido das participantes da pesquisa e de sua mãe ou responsável. Logo após, as participantes responderam ao instrumento, em um só encontro.

Foi utilizado um questionário semi-estruturado, validado para a presente pesquisa a partir do estudo-piloto, contendo questões que investigavam variáveis sócio-demográficas (idade, grau de escolaridade, renda familiar, moradia, estado civil e religião) e variáveis biológicas, tais como a idade da menarca, idade ginecológica, idade da primeira gravidez, número de gestações levadas a termo ou interrompidas, número de filhos vivos, realização de planejamento familiar, conhecimento e uso de métodos contraceptivos. Estas duas últimas variáveis acerca do conhecimento 
e uso de métodos contraceptivos foram investigadas através de questões abertas em que era solicitado que a adolescente citasse os métodos contraceptivos que conhece, qual utilizava e há quanto tempo. Foi utilizada a análise de frequência de respostas para avaliação destas variáveis.

A presente investigação atendeu a todas as exigências do Comitê de Ética em Pesquisa da Universidade Federal do Rio Grande do Norte e foi aprovada por obedecer aos procedimentos preconizados pela Resolução no 196/96 do Conselho Nacional de Saúde para pesquisas com seres humanos. O questionário utilizado foi analisado e os dados obtidos foram processados e trabalhados através da Estatística Descritiva com frequência de respostas e medidas de tendência central.

\section{Resultados}

As 50 adolescentes entrevistadas com repetição de gravidez apresentam idade média de 17 anos (com desvio-padrão de 1 ano de idade). Ressalta-se os maiores percentuais dentre as categorias observadas quanto ao perfil psicossocial do grupo estudado, a saber: $62 \%$ estão unidas estavelmente, $56 \%$ adotam o catolicismo como religião e $86 \%$ apresentam o Ensino Fundamental Incompleto como escolaridade atual (Tabela 01), sendo que atualmente $82 \%$ (41) das adolescentes não estudam. A maioria das adolescentes não trabalha $(84 \%)$.

Tabela 01: Perfil sócio-demográfico (estado civil, religião e escolaridade) das adolescentes com repetição de gravidez usuárias das enfermarias de Maternidade Escola J anuário Cicco, em Natal/RN/BR.

\begin{tabular}{|c|c|c|c|c|c|c|c|c|}
\hline \multicolumn{3}{|c|}{ Estado Civil } & \multicolumn{3}{|c|}{ Religião } & \multicolumn{3}{|c|}{ Escolaridade } \\
\hline Categoria & $\mathbf{n}$ & Frequência & Categoria & n & Frequência & Categoria & n & Frequência \\
\hline $\begin{array}{l}\text { União } \\
\text { Estável }\end{array}$ & 31 & $62 \%$ & Católica & 28 & $56 \%$ & Fundamental & 43 & $86 \%$ \\
\hline Solteira & 13 & $26 \%$ & Evangélica & 6 & $12 \%$ & & & \\
\hline Casada & 4 & $8 \%$ & Não & 16 & 330 & Médi & 7 & $140 \%$ \\
\hline Divorciada & 2 & $4 \%$ & possui & 16 & & Incompleto & 7 & \\
\hline TOTAL & 50 & $100 \%$ & TOTAL & 50 & $100 \%$ & TOTAL & 50 & $100 \%$ \\
\hline
\end{tabular}

Quanto à renda familiar, 68\% da amostra possui entre 1 e 3 saláriosmínimos (SM), provenientes, em sua maioria, do trabalho de suas mães e de seus companheiros, ou de pensões. Esta renda sustenta em média 5 pessoas em suas casas, que correspondem a famílias geracionais em $54 \%$ da amostra, constituída normalmente pela adolescente, seu companheiro e filho(s). Já nas famílias multigeracionais, que correspondem a $47 \%$ da amostra, as adolescentes em geral convivem com uma geração que normalmente inclui os pais ou os sogros delas (Tabela 02). 
Tabela 02: Perfil sócio-demográfico (renda familiar, núcleo familiar, cômodos por domicílio) das adolescentes com repetição de gravidez usuárias das enfermarias de Maternidade Escola Januário Cicco, em Natal/RN/BR.

\begin{tabular}{|c|c|c|c|c|c|c|c|c|}
\hline \multicolumn{3}{|c|}{ Renda familiar } & \multicolumn{3}{|c|}{ Núcleo familiar } & \multicolumn{3}{|c|}{ Cômodos por domicílio } \\
\hline Categoria & $\mathbf{n}$ & Frequência & Categoria & $\mathbf{n}$ & Frequência & Categoria & $\mathbf{n}$ & Frequência \\
\hline $\begin{array}{l}\text { Menos de } \\
1 \text { SM }\end{array}$ & 8 & $16 \%$ & & 27 & $54 \%$ & 1 a 3 & 13 & $26 \%$ \\
\hline 1 a $3 \mathrm{SM}$ & 34 & $68 \%$ & & & & 4 a 6 & 28 & 56 \\
\hline $\begin{array}{l}4 \text { a } 6 \text { SM } \\
\text { Não sabe }\end{array}$ & $\begin{array}{l}3 \\
5\end{array}$ & $\begin{array}{l}6 \% \\
10 \%\end{array}$ & $\begin{array}{c}\text { Família } \\
\text { Multigeracional }\end{array}$ & 23 & $46 \%$ & $\underset{7}{\text { Acima de }}$ & 9 & $18 \%$ \\
\hline TOTAL & 50 & $100 \%$ & TOTAL & 50 & $100 \%$ & TOTAL & 50 & $100 \%$ \\
\hline
\end{tabular}

Quanto às variáveis biológicas, as participantes desse estudo tiveram sua menarca, em média, aos 11 anos de idade, e sua primeira relação sexual, em média, aos 13 anos. As adolescentes entrevistadas já engravidaram, em média, duas vezes na adolescência, ocorrendo a primeira gravidez por volta dos 14 anos de idade (Tabela 03). Quanto ao número de gestações na adolescência, $66 \%$ (33) das adolescentes engravidaram duas vezes, $32 \%$ (16) engravidaram três vezes e uma delas $(2 \%)$ já havia engravidado cinco vezes.

Tabela 03: Perfil das variáveis biológicas das adolescentes com repetição de gravidez usuárias das enfermarias de Maternidade Escola Lanuário Cicco, em Natal/RN/BR.

\begin{tabular}{|c|c|c|c|c|c|c|}
\hline & \multicolumn{2}{|c|}{ Menarca } & \multicolumn{2}{c|}{ Primeira relação sexual } & \multicolumn{2}{c|}{ Primeira gravidez } \\
\hline Categorias & n & Frequência & n & Frequência & n & Frequência \\
\hline $\begin{array}{c}\text { Menos de } \\
\mathbf{1 2} \text { anos }\end{array}$ & 22 & $44 \%$ & 4 & $8 \%$ & 0 & 0 \\
\hline $\begin{array}{c}\mathbf{1 2} \text { a 14 } \\
\text { anos }\end{array}$ & 26 & $52 \%$ & 35 & $70 \%$ & 25 & $50 \%$ \\
\hline $\begin{array}{c}\text { Acima de } \\
\text { 14 anos }\end{array}$ & 1 & $2 \%$ & 11 & $22 \%$ & 25 & $50 \%$ \\
\hline $\begin{array}{c}\text { Não } \\
\text { lembra }\end{array}$ & 1 & $2 \%$ & 0 & 0 & 0 & 0 \\
\hline TOTAL & $\mathbf{5 0}$ & $\mathbf{1 0 0} \%$ & $\mathbf{5 0}$ & $\mathbf{1 0 0} \%$ & $\mathbf{5 0}$ & $\mathbf{1 0 0} \%$ \\
\hline
\end{tabular}

Quando questionadas sobre suas gestações, $96 \%$ (48) das adolescentes possuem filhos vivos. Quanto à idade destes, 53,1\% (43) da amostra possui filhos vivos com idade até 1 ano de vida, $34,6 \%$ (28) possui filhos com idade entre 1 e 3 anos, e 12,3\% (10) entre 4 e 6 anos de idade. Entretanto, algumas dessas gestações não foram levadas a termo, identificando-se a ocorrência de 26 abortos dentre $40 \%$ (20) das adolescentes. Destes abortos, $73 \%$ (19) foram naturais e $27 \%$ (7) foram provocados. Ainda com relação ao planejamento, $88 \%$ (44) das adolescentes não participaram de programas de planejamento familiar.

As adolescentes com repetição de gravidez que participaram desta investigação conhecem em média 03 (três) métodos 
Anticoncepcionais (MAC). Através desse conhecimento revelado espontaneamente, a pílula foi o método mais citado, uma vez que $96 \%$ (48) das adolescentes revelam conhecer esse método hormonal oral. Seguindo a ordem de conhecimento, 92\% (46) das adolescentes citaram a camisinha como MAC, 60\% (30) a injeção, 44\% (22) o DIU, $10 \%$ (5) o diafragma, $4 \%$ (2) a laqueadura, $2 \%$ (1) um "remédio natural para não engravidar", e $1(2,2 \%)$ adolescente referiu não conhecer método contraceptivo algum.

Quando questionadas sobre o uso de algum método contraceptivo na sua primeira relação sexual, $54 \%$ (27) das adolescentes do presente estudo não utilizaram método algum, em oposição às $46 \%$ (23) que usaram. Destas, $78,3 \%$ (18) fizeram uso da camisinha em sua primeira experiência sexual, $17,4 \%$ (4) da pílula, e 1 (4,8\%) adolescente utilizou a pílula e a camisinha associadamente.

Ao serem questionadas sobre o uso de algum método contraceptivo antes de sua atual ou última gravidez, $56 \%$ (28) das adolescentes com repetição de gravidez afirmaram estar usando algum MAC. Dentre estas, os mais usados foram a pílula por $68 \%$ (19) delas, a camisinha por $25 \%$ (7) delas e a injeção hormonal por $7 \%$ (2) das adolescentes. Esse uso aconteceu entre 1 e 2 anos antes da atual ou última concepção para 50\% (14) das adolescentes, entre 1 e 6 meses para $29 \%$ (8) delas, entre 3 e 4 anos para $13 \%$ (4) das adolescentes que usavam algum MAC antes de sua atual ou última gravidez, 1 (4\%) já estava grávida e 1 (4\%) não lembra desde quando usava o MAC.

\section{Discussão}

Nos estudos sobre a gravidez na adolescência, costuma-se dividir a fase da adolescência em dois períodos com características e riscos próprios: dos 12 aos 14 e dos 15 aos 18 anos de idade. No caso da ocorrência de gravidez, nesta primeira fase, o risco é maior tanto para mãe quanto para o bebê. No entanto, evidencia-se um aumento no número de partos nesta faixa etária, o que preocupa, cada vez mais, os pesquisadores e profissionais de saúde (CARNIEL et al., 2006).

Por diversos fatores já descritos anteriormente, a gravidez na adolescência é um fenômeno multifatorial que merece a atenção de toda a sociedade. É um problema de saúde pública que atinge todas as classes sociais, mas que parece ter um impacto maior sobre as adolescentes dos mais baixos níveis sócio-econômicos e suas famílias (AQUINO et al., 2003; BARALDI et al., 2007; CARNIEL et al., 2006; GAMA; SZWARCWALD; LEAL, 2002). E essa difícil realidade deve ser característica para as adolescentes da presente investigação, 
pertencentes a classes sociais de baixo poder aquisitivo, conforme se pode observar através de seus dados sócio-econômicos.

Como outro fator agravante e associado ao aumento da fecundidade na adolescência, tem-se o fato de que estas adolescentes apresentam uma escolaridade que não acompanha sua faixa etária, além de que, no momento da pesquisa, não frequentavam a escola, o que pode acarretar prejuízos tanto em sua formação pessoal quanto profissional, bem como impedir a construção de projetos de vida. Alguns autores consideram exatamente o abandono escolar ou a defasagem nos estudos como um dos fatores que caracteriza a gravidez na adolescência como um problema de saúde pública no Brasil. Isto porque a gravidez nessa faixa etária não traz apenas consequências negativas em nível biológico para a saúde da mãe e do bebê, mas, principalmente, acarreta efeitos negativos à inserção da adolescente no mercado de trabalho, prejudicando suas condições de estudo e intensificando as dependências familiares, advindo, assim, consequências desfavoráveis na perspectiva de vida e de trabalho (MICHELAZZO et al., 2004; SABROZA et al., 2004).

A interrupção da escolarização observada na amostra pode ainda perpetuar a tendência à pobreza, com acentuação dos riscos psicossociais aos quais mães adolescentes e seus filhos estão submetidos (AQUINO et al., 2003; BARALDI et al., 2007; CARNIEL et al., 2006; GAMA et al., 2002; SOUZA et al., 2001).

A ocorrência de gravidez no contexto de relacionamentos estáveis e de morar com famílias geracionais são dados apresentados pela maioria das adolescentes participantes desta investigação, e também de outros estudos (AQUINO et al., 2003; CARNIEL et al., 2006; CHALEM et al., 2007; DIAS; AQUINO, 2006), o que se configura como um aspecto positivo para o desenvolvimento saudável da adolescente e de seu filho, na medida em que o companheiro pode apoiar econômica e psicologicamente a sua companheira adolescente (CARNIEL et al., 2006).

Percebe-se ainda que a precocidade da menarca e do início da vida sexual (ambos abaixo dos 14 anos de idade no presente estudo) tem tornado as adolescentes vulneráveis à repetição de gravidez na adolescência, conforme se identifica entre as adolescentes entrevistadas. Estas adolescentes também se caracterizam por terem engravidado, em sua maioria, no mínimo duas vezes. Apresentam uma diferença média de 1 ano entre a primeira relação sexual e a primeira gestação, e possuem filhos vivos, convivendo com os mesmos, e com ocorrência de abortos naturais na maioria dos casos. Tais resultados estão de acordo com pesquisas anteriores realizadas também com adolescentes com repetição de gravidez (CHALEM et al., 2007; GAMA, S. G. N.; SZWARCWALD, C. L.; LEAL, M. C. , 2002; SOUZA et al., 2001; VIEIRA et al., 2005). 
O aborto entre adolescente revela uma lacuna entre o conhecimento e a utilização de métodos anticoncepcional, sendo, por vezes, utilizado como método contraceptivo. Este quadro poderia ser revertido a partir de uma adequada educação em saúde, podendo diminuir a morbimortalidade materna relativa a essa faixa etária (SCHOR, N.; LÓPEZ, A. F., 1990).

A ausência de planejamento familiar eficaz também pode proporcionar o processo de repetição da gravidez e pode ser decorrente da precariedade e deficiência dos serviços de saúde oferecidos às adolescentes, que, por vezes, não contam com uma equipe multidisciplinar motivada e capacitada para trabalhar e atender as necessidades destas jovens (BARALDI et al., 2007; CARNIEL et al., 2006).

Tendo em vista o grande número de MAC existente atualmente, percebe-se que as adolescentes da amostra conhecem um pequeno número de métodos que previnem a gravidez e que a camisinha não foi vislumbrada como MAC por todas as adolescentes, embora exista uma grande divulgação desse método na mídia, não só como método contraceptivo, mas também como recurso para evitar as DSTs. Percebe-se ainda a presença de uma adolescente que espontaneamente não revelou conhecer MAC algum, 0 que pode indicar a falta de informação sobre a contracepção ou a não compreensão da questão que lhe foi feita. Tais resultados corroboram com os dados encontrados nos estudos realizados por Belo e Pinto e Silva (2004) e por Vieira et al. (2005), os quais acreditam que, apesar do conhecimento ser um elemento necessário para o uso, não existe uma associação entre os níveis de conhecimento e as taxas de utilização, em virtude, dentre outras razões, da imaturidade psicoemocional característica da adolescência, do medo de ser descoberta usando algum MAC, do temor de que os métodos possam prejudicar a sua saúde, de não saber usá-los adequadamente, da dificuldade em obtê-los ou do desejo em engravidar.

Dessa forma, percebe-se que a informação e o conhecimento de MAC podem ser fundamentais para a conscientização de seu uso, tendo em vista que as adolescentes conhecem os MAC e geralmente sabem como obtê-los, embora isso não garanta o seu uso efetivo e seguro (LONGO, 2002).

No estudo de Lima et al. (2004), 68,4\% das adolescentes dizem ter feito uso de método contraceptivo na primeira relação sexual, correspondendo a um índice mais alto do encontrado na presente investigação, na qual a maioria não fez uso de método algum nesse momento. As principais razões levantadas por Lima et al. (2004) para não evitar filhos foram a falta de expectativa quanto à relação sexual e à gravidez, e o desejo de engravidar. Entretanto, houve uma concordância quanto ao MAC utilizado na primeira relação, uma vez 
que a camisinha foi utilizada por $87,5 \%$ das adolescentes na pesquisa de Lima et al. (2004).

Um percentual mais próximo da presente investigação foi o encontrado no estudo de Aquino et al. (2003), no qual 52,5\% das adolescentes revelaram o uso de contracepção na primeira relação, sendo o preservativo masculino (a camisinha) o mais usado por essas adolescentes. É interessante ressaltar a relação inversa existente entre o uso de contracepção na iniciação sexual e a gravidez na adolescência, uma vez que a gravidez na adolescência foi mais frequente $(63,9 \%)$ entre mulheres que disseram não ter usado método algum na sua primeira relação sexual. Esse uso inicial também depende do vínculo que a adolescente tem com o seu parceiro de relação e sua capacidade de negociação quanto à escolha do método contraceptivo a ser usado pelo casal (AQUINO et al., 2003). O percentual encontrado no estudo de Belo \& Pinto e Silva (2004) também foi próximo ao do presente estudo.

$\mathrm{O}$ estudo de Vieira et al. (2005) evidencia que pesquisas realizadas na América Latina têm mostrado que menos de $20 \%$ dos homens e menos de $15 \%$ das mulheres usam algum método anticoncepcional na primeira relação sexual. Isto indica um percentual muito baixo que pode ser associado aos altos índices de fecundidade existentes em países dessa região, assim como o encontrado no presente estudo, no qual a maioria das adolescentes (54\%) não fez uso de MAC algum em sua primeira relação. Esses autores citam ainda outro estudo no qual apenas $8 \%$ dos jovens referiram ter se protegido na primeira experiência sexual, sendo o preservativo masculino (a camisinha) $(66,7 \%)$ e a pílula $(36,7 \%)$ os mais utilizados nesse momento, corroborando com os dados obtidos na investigação aqui apresentada.

Essas questões referentes à primeira experiência sexual das adolescentes apresentam sua relevância tendo em vista o caráter preditor desta para com as práticas contraceptivas subseqüentes. Um exemplo dessa associação é que as mulheres que usaram MAC na primeira relação sexual têm cerca de duas vezes mais a chance de terem usado MAC na última relação do que as que não utilizaram (LONGO, 2002).

Coard; Nitz; Felive (2002) apontam para uma associação entre o MAC e o seu uso atual pelas adolescentes e a ocorrência de repetição de gravidez na adolescência tanto nos 12 meses iniciais após o seu último parto ou aborto, quanto entre 12 e 24 meses após esse período. O tempo, portanto, entre o último parto ou aborto e o início de contracepção apresenta uma relação com a repetição de gravidez. Este estudo também apóia os dados da presente pesquisa, na qual a maioria das adolescentes fazia uso de algum MAC (56\%) e $50 \%$ destas também iniciaram este uso entre 12 e 24 meses após o último 
parto ou aborto - período no qual se insere o tempo de ocorrência de repetição de gravidez.

Das mães adolescentes estudadas por Coard et al. (2002), 76\% que relataram usar MAC consistentemente no período pós-parto imediato (entre 1 e 16 semanas do último parto) não tiveram repetição de gravidez após 24 meses de pós-parto. Além disso, os autores perceberam que as mães adolescentes que usaram contracepção de longa ação e no pós-parto imediato tiveram uma taxa de repetição de gravidez de $4,9 \%$ no 1 을 ano de pós-parto, comparado com $25 \%$ das adolescentes que usaram contraceptivos orais e $46 \%$ das que usavam camisinha. Este resultado também está de acordo com os dados encontrados por esta pesquisa, na qual $44 \%$ das adolescentes com repetição de gravidez não faziam uso de MAC algum antes da atual gestação, e as que faziam apenas $2 \%$ utilizam contraceptivo injetável. Com isso, evidencia-se que o uso de contraceptivos injetáveis e de longa duração (PERSONA et al., 2004), um planejamento familiar iniciado ainda no período do puerpério e o acompanhamento do uso contraceptivo durante os primeiros 24 meses de pós-parto são importantes e recomendados para a prevenção de gravidez entre mães adolescentes (COARD et al., 2002).

Outro fator relacionado à repetição de gravidez na adolescência é o baixo uso de camisinha (CARNIEL et al., 2006; PERSONA, L.; SHIMO, A. K. K.; TARALLO, M. C., 2004), o que está em concordância com os achados da presente pesquisa que revela uma pequena utilização da camisinha após o último parto ou aborto entre as adolescentes investigadas. Aliás, a baixa incidência dos métodos contraceptivos em geral é considerada um motivo para a gravidez na adolescência (SOUZA et al., 2001), como em um estudo de Chalem et al. (2007) que identificou que a minoria das adolescentes menores de 16 anos $(20,9 \%)$ e maiores de 16 anos $(25,6 \%)$ faziam uso de algum método contraceptivo quando engravidou.

Destaca-se, portanto, o não uso de MAC após o último parto ou aborto das adolescentes com repetição de gravidez que participaram da pesquisa mesmo considerando o fato de que todas são multigestas e que ter um bebê poderia servir como um despertar para as adolescentes, motivando-as para engajar-se ativamente em um planejamento familiar e comportamento contraceptivo efetivo (KERSHAW et al., 2003). A presença dessas adolescentes que não faziam uso de MAC algum pode ser explicada pelo desejo de ter filhos, o desconhecimento de MAC, a ocorrência de erro na utilização do contraceptivo ou o seu uso inconsistente, características próprias da adolescência, como o pensamento mágico associado a outros fatores psicossociais e comunitários (BELO; PINTO E SILVA, 2004; COARD, S. I.; NITZ, K.; FELIVE, M. E., 2002; LIMA et al., 2004). Quando não há o desejo em engravidar, esse não uso de MAC indica 
um comportamento de risco, pois a relação sexual desprotegida pode resultar em uma gravidez não desejada e em DSTs (LONGO, 2002).

Em seu estudo, Persona et al. (2004) encontraram que a pílula foi o método contraceptivo mais utilizado pelas adolescentes $(55,5 \%)$ durante o período entre o último parto ou aborto e o início da gestação atual, seguido pela camisinha $(27,8 \%)$ e pelo hormônio injetável (22,2\%). A ausência de MAC apareceu em $11,1 \%$ das adolescentes. Essa sequência é a mesma encontrada na presente investigação, embora os percentuais de não uso e de uso da pílula tenham sido mais altos, e os de uso da camisinha e da injeção mais baixos.

Outro estudo (KERSHAW et al., 2003) aponta para o fato de que a maioria das jovens mães usa contraceptivos após o último parto ou aborto e o MAC escolhido geralmente é o hormonal. No entanto, identifica-se um uso mais consistente nos 6 meses após o pós-parto, com um significante decréscimo do uso hormonal após esses 6 meses iniciais. Esse mesmo estudo evidenciou ainda que as mães adolescentes são 2,3 vezes mais prováveis para usar consistentemente camisinha e contraceptivo hormonal juntos, e 1,8 vezes mais prováveis para usar consistentemente somente 0 contraceptivo hormonal que as adolescentes que não foram mães.

Faz-se necessário, portanto, um trabalho educativo prévio e, se possível, durante o internamento resultante de gravidez, parto ou puerpério da adolescente, um encaminhamento para programas de planejamento familiar, além de um acompanhamento efetivo dessas adolescentes no pós-parto tardio e da realização de esclarecimentos sobre sexualidade, contracepção, riscos e complicações que resultem de práticas contraceptivas não-médicas e não-seguras (BARRERA; PICK, 2006; COARD, S. I.; NITZ, K.; FELIVE, M. E., 2002; SOUZA et al., 2001; LONGO, 2002).

Nesta perspectiva, os dados obtidos com a presente investigação parecem confirmar questões discutidas anteriormente que chamam atenção para os diversos fatores associados à repetição de gravidez na adolescência, tais como a baixa escolaridade estando relacionada à repetição de gravidez, assim como a presença de relacionamentos estáveis, o uso inconsistente de métodos contraceptivos e, principalmente, o desejo de ser mãe como um projeto de vida dessas adolescentes. Assim sendo, salienta-se a necessidade de execução imediata de intervenções voltadas para as demandas já conhecidas e identificadas junto às próprias adolescentes e jovens que carecem de uma assistência eficaz e eficiente. 


\section{Referências}

AQUINO, E. M. L.; HEILBORN, M. L.; KNAUTH, D.; BOZON, M.; ALMEIDA, M. C.; ARAÚJO, J.; MENEZES, G. Adolescência e reprodução no Brasil: A heterogeneidade dos perfis sociais. Cadernos de Saúde Pública, Rio de Janeiro, v. 19, n. 2, p. 377388, 2003.

BARALDI, A. C. P.; DAUD, Z. P.; ALMEIDA, A. M.; GOMES, F. A.; NAKANO, A. M. S. Gravidez na adolescência: estudo comparativo das usuárias das maternidades públicas e privadas. Revista LatinoAmericana de Enfermagem, Ribeirão Preto, v. 15, p. 799-805, setembro/outubro 2007.

BARRERA, C. P.; PICK, S. Conducta sexual protegida en adolescentes mexicanos. I nteramerican J ournal of Psychology, Porto Alegre, v. 40, n. 3, p. 333-340, 2006.

BELO, M. A. V.; PINTO E SILVA, J. L. Conhecimento, atitudes e prática sobre métodos anticoncepcionais entre adolescentes gestantes. Revista de Saúde Pública, São Paulo, v. 38, n. 4, p. 479-487, 2004.

CÂMARA, S. G.; SARRIERA, J. C.; CARLOTTO, M. S. Predictores de conductas sexuales de riesgo entre adolescentes. Interamerican J ournal of Psychology, Porto Alegre, v. 41, n. 2, p. 161-166, 2007. CARNIEL, E. F.; ZANOLLI, M. L.; ALMEIDA, C. A. A.; MORCILLO, A. M. Características das mães adolescentes e de seus recém-nascidos e fatores de risco para a gravidez na adolescência em Campinas, SP, Brasil. Revista Brasileira de Saúde Materno I nfantil, Recife, v. 6, n. 4, p. 419-426, outubro/dezembro 2006.

CHALEM, E.; MITSUHIRO, S. S.; FERRI, C. P.; BARROS, M. C. M.; GUINSBURG, R.; LARANJEIRA, R. Gravidez na adolescência: perfil sócio-demográfico e comportamental de uma população da periferia de São Paulo, Brasil. Cadernos de Saúde Pública, Rio de Janeiro, v. 23, n. 1, p. 177-186, janeiro 2007.

COARD, S. I.; NITZ, K.; FELIVE, M. E. Repeat pregnancy among urban adolescents: Sociodemographic, family, and health factors. Adolescence, New York, v. 35, p. 193-200, 2002.

DIAS, A. B.; AQUINO, E. M. L. Maternidade e paternidade na adolescência: algumas constatações em três cidades do Brasil. Cadernos de Saúde Pública, Rio de Janeiro, v. 22, n. 7, p. 14471458, julho 2006.

GAMA, S. G. N.; SZWARCWALD, C. L.; LEAL, M. C. Experiência de gravidez na adolescência, fatores associados e resultados perinatais entre puérperas de baixa-renda. Cadernos de Saúde Pública, Rio de Janeiro, v. 18, n. 1, p. 153-161, janeiro/fevereiro 2002.

GODINHO, R. A.; SCHELP, J. R. B.; PARADA, C. M. G. L.; BERTONCELLO, N. M. F. Adolescentes e grávidas: onde buscam 
apoio? Revista Latino-Americana de Enfermagem, Ribeirão Preto, v. 8, n. 2, p. 25-32, abril 2000.

GOLDENBERG, P.; FIGUEIREDO, M. C. T.; SILVA, R. S. Gravidez na adolescência, pré-natal e resultados perinatais em Montes Claros, Minas Gerais, Brasil. Cadernos de Saúde Pública, Rio de Janeiro, v. 21, n. 4, p. 1077-1086, julho/agosto 2005.

KERSHAW, T. S.; NICCOLAI, L. M.; ICKOVICS, J. R.; LEWIS, J. B.; MEADE, C. S.; ETHIER, K. A. Short and long-term impact of adolescent pregnancy on postpartum contraceptive use: implications for prevention of repeat pregnancy. J ournal of Adolescent Health, San Francisco, California, v. 33, p. 359-368, 2003.

LIMA, C. T. B.; FELICIANO, K. V. O.; CARVALHO, M. F. S.; SOUZA, A. P. P.; MENABÓ, J. B. C.; RAMOS, L. S.; CANINDÉ, L. F.; KOVACS, M. H. Percepções e práticas de adolescentes grávidas e de familiares em relação à gestação. Revista Brasileira de Saúde Materno I nfantil, Recife, v. 4, n. 1, p. 71-83, 2004.

LONGO, L. A. F. B. Juventude e contracepção: um estudo dos fatores que influenciam o comportamento contraceptivo das jovens brasileiras de 15 a 24 anos. Revista Brasileira de Estudos da População, Rio de Janeiro, v. 19, n. 2, p. 230-247, julho/dezembro 2002.

MALDONADO, M. T. P. Psicologia da gravidez: parto e puerpério. 14 ed. São Paulo (SP): Saraiva, 1997.

MALDONADO, M. T. Psicologia da gravidez. 16 ed. São Paulo: Saraiva; 2002.

MATERNIDADE ESCOLA JANUÁRIO CICCO. In: WIKIPÉDIA, a enciclopédia livre. Flórida: Wikimedia Foundation, 2010. Disponível em:

$<$ http://pt. wikipedia.org/w/index.php?title=Maternidade_Escola_J anu \%C3\%A1rio_Cicco\&oldid=19784394>. Acesso em: 17 nov. 2010.

MICHELAZZŌ, D.; YAZLLE, M. E. H. D.; MENDES, M. C.; PATTA, M. C.; ROCHA, J. S. Y.; MOURA, M. D. Indicadores sociais de grávidas adolescentes: Estudo caso-controle. Revista Brasileira de Ginecologia e Obstetrícia, Rio de Janeiro, v. 26, n. 8, p. 633-639, 2004.

MONTEIRO, I. P. Aspectos sociais e reprodutivos das mulheres submetidas à laqueadura tubária na Maternidade Municipal Mãe Esperança em Porto Velho/ RO. 2009. 80f. Dissertação (Mestrado em Ciências da Saúde) - Faculdade de Ciências da Saúde, Universidade de Brasília, Brasília.

OLIVEIRA, N. R. Perfis de grávidas e mães adolescentes: estudo psicossocial de adolescentes usuárias de um serviço público de pré-natal e maternidade. 1999. 170f. Tese (Doutorado Psicologia Social). Instituto de Psicologia, Universidade de São Paulo, São Paulo. 
PANTOJA, A. L. N. Ser alguém na vida: uma análise sócioantropológica da gravidez/maternidade na adolescência, em Belém do Pará, Brasil. Cadernos de Saúde Pública, Rio de Janeiro, v.19, n. 2, p. 335-343, 2003.

PERSONA, L.; SHIMO, A. K. K.; TARALLO, M. C. Perfil de adolescentes com repetição da gravidez atendidas num ambulatório de pré-natal. Revista Latino-Americana de Enfermagem, Ribeirão Preto, v. 12, n. 5, p. 745-750, setembro/outubro 2004.

SABROZA, A. R.; LEAL, M. C.; GAMA, S. G. N.; COSTA, J. V. Perfil sócio-demográfico e psicossocial de puérperas adolescentes do município do Rio de Janeiro, Brasil - 1999-2001. Cadernos de Saúde Pública, Rio de Janeiro, v. 20, n. 1, p. 112-120, 2004.

SCHOR, N.; LÓPEZ, A. F. Adolescência e anticoncepção: 1. Estudo de conhecimento e uso em puérperas internadas por parto ou aborto. Revista Saúde Pública, São Paulo, v. 24, n. 6, p. 506-511, dezembro 1990.

SOUZA, V. L. C.; CORRÊA, M. S. M.; SOUZA, S. L.; BESERRA, M. A. O aborto entre adolescentes. Revista Latino-Americana de Enfermagem, Ribeirão Preto, v. 9, n. 2, p. 42-47, março 2001.

VIEIRA, L. M.; SAES, S. O.; DÓRIA, A. A. B.; GOLDBERG, T. B. L. Reflexões sobre a anticoncepção na adolescência no Brasil. Revista Brasileira de Saúde Materno I nfantil, Recife, v. 6, n. 1, p. 135140, janeiro/março 2005.

\section{Endereço para correspondência \\ Camomila Lira Ferreira}

Rua Arapuá, no 263, Nordeste, CEP 59042-430, Natal-RN, Brasil

Endereço eletrônico: camomilapsi@yahoo.com.br

\section{Liliane Pereira Braga}

Rua J oão Celso Filho, no 2531, Bairro Lagoa Nova, CEP 59064-320, Natal-RN, Brasil Endereço eletrônico: lilibraga@gmail.com

\section{Ádala Nayana de Sousa Mata}

Rua Dr. Miguel Couto, número 86, Bairro Pitimbu, CEP 59060-020, Natal-RN, Brasil Endereço eletrônico: adalamata@gmail.com

\section{Caroline Araújo Lemos}

Rua Adeodato José dos Reis, no 1100, Apt. 1403A, Bairro Nova Parnamirim, CEP 59152-820, Parnamirim-RN, Brasil

Endereço eletrônico: carolpsilemos@yahoo.com.br

Eulália Maria Chaves Maia

Rua Seridó, 754, Apto. 902, Petrópolis, CEP 59020-010, Natal-RN, Brasil

Endereço eletrônico: eulalia.maia@yahoo.com.br

Recebido em: 04/03/2010

Reformulado em: 18/11/2010

Aceito para publicação em: 13/12/2010

Acompanhamento do processo editorial: Ariane Patricia Ewald 


\section{Notas}

* Doutoranda pelo Programa de Pós-Graduação em Ciências da Saúde (PPGCSa) da Universidade Federal do Rio Grande do Norte (UFRN), Pesquisadora Voluntária do Grupo de Estudos: Psicologia e Saúde (GEPS) do Departamento de Psicologia da UFRN; Natal/RN, Brasil; Psicóloga, Especialização em Psicologia da Saúde: Desenvolvimento e Hospitalização pela UFRN.

**Mestranda pelo Programa de Pós-Graduação em Psicologia da UFRN, Pesquisadora Voluntária do GEPS; Natal/RN, Brasil; Psicóloga, Especialização em Psicologia da Saúde: Desenvolvimento e Hospitalização pela UFRN.

*** Docente da Faculdade Maurício de Nassau; Mestre pelo PPGCSa da UFRN, Pesquisadora Voluntária do GEPS; Natal/RN, Brasil; Psicóloga, Especialização em Psicologia da Saúde: Desenvolvimento e Hospitalização pela UFRN.

****Docente do Curso de Psicologia da Universidade Potiguar; Mestre pelo Programa de Pós-Graduação em Psicologia da UFRN, Pesquisadora Voluntária do Laboratório de Pesquisa e Extensão em Neuropsicologia do Departamento de Psicologia da UFRN; Natal/RN, Brasil; Psicóloga, Especialização em Psicologia da Saúde: Desenvolvimento e Hospitalização pela UFRN.

***** Professora Associada do Departamento de Psicologia da UFRN, Orientadora do PPGCSa e do Programa de Pós-Graduação em Psicologia da UFRN, Coordenadora do GEPS; Natal/RN, Brasil; Psicóloga, Doutorado em Psicologia Clínica pela Universidade de São Paulo. 